\title{
Medicamentos e amamentação: atualização e revisão aplicadas à clínica materno-infantil
}

\author{
Medicines and breastfeeding: update and revision applied to mother and baby care
}

Roberto Gomes Chaves ${ }^{1}$, Joel Alves Lamounier ${ }^{2}$, Cibele Comini César ${ }^{3}$

\section{RESUMO}

Objetivo: Realizar revisão bibliográfica sobre o uso de medicamentos durante o período de lactação.

Fontes de dados: Artigos nos bancos de dados eletrônicos Pubmed, Medline, Lilacs e SciELO nos últimos dez anos, nas línguas portuguesa, inglesa e espanhola, utilizando os descritores "aleitamento materno", "lactação", "leite humano" e "uso de medicamentos". Também foram consultados livros, textos recentes e artigos considerados relevantes para realização dessa revisão.

Síntese dos dados: Os fármacos foram classificados em tabelas, conforme o risco para uso na lactação. Há carência de informações sobre a segurança de muitas medicações durante a amamentação. Dentre os fármacos com informações sobre segurança para uso na lactação, a maior parte é considerada segura e poucos são contra-indicados. Alguns merecem preocupação devido ao risco de redução da lactogênese ou produção de efeitos adversos sobre o lactente. $\mathrm{O}$ uso de galactagogos está restrito a situações especiais, pouco freqüentes na prática clínica.

Conclusões: A freqüente necessidade do uso de medicamentos na lactação deve ser encarada com preocupação devido à conhecida relação entre o uso de fármacos e o desmame. $\mathrm{O}$ princípio fundamental da prescrição de medicamentos para lactantes baseia-se na avaliação dos riscos e dos benefícios. Neste momento, o conhecimento de características farmacológicas e a consulta a publicações atualizadas sobre o tema são importantes instrumentos na escolha do fármaco. Uma avaliação cuidadosa quase sempre permite compatibilizar o tratamento medicamentoso com a amamentação.

Palavras-chave: aleitamento materno; lactação; medicamentos; drogas.

\section{ABSTRACT}

Objective: To present an updated review about the use of medication during breastfeeding.

Data sources: Papers from electronic databases Pubmed, Medline, Lilacs and SciELO, from the last ten years in Portuguese, English and Spanish, were retrieved using the key-words "breastfeeding", "lactation", "human milk" and "use of medication". Recent material from textbooks and relevant papers were also used for this review.

Data synthesis: Medicines were classified according to the risk associated to lactation use. There is a lack of information about safety of a vast number of medicines by the nursing mothers. Amongst the medicines containing safety information for lactation use, most are considered safe and few are not recommended. Some drugs raise concerns regarding their effect in the reduction of milk production or in causing adverse effects in the breastfed infants. The use of stimulants of milk production is restricted to special situations, not common in the clinical practice.

Conclusions: The frequent need of medication during lactation must raise some concern due to the well-known relationship between its use and weaning. Medical prescription for nursing mothers should be evaluated by risks versus benefits analysis. Currently, the knowledge of pharmacological characteristics of the available medication and the continuous updating about this subject are important tools to choose the medication. A careful assessment allows generally compatibility between medical treatment and breastfeeding.

Key-words: breast feeding; lactation; medication; drugs.

\footnotetext{
'Mestre e doutorando em Ciências da Saúde e Saúde da Criança e do Adolescente pela Universidade Federal de Minas Gerais (UFMG) e professor auxiliar de Farmacologia da Universidade de Itaúna

2Doutor em Saúde Pública pela University of Califórnia, Los Angeles, e professor adjunto da Faculdade de Medicina da UFMG

${ }^{3}$ Doutora em Estatística pela UFMG e professora adjunta da Faculdade de Ciências Exatas da UFMG
}

Endereço para correspondência

Roberto Gomes Chaves

Rua Mariângela Medeiros, 50 - Centro

CEP 35680-457 - Itaúna/MG

E-mail: robertogchaves@uol.com.br

Recebido em: 2/4/2007

Aprovado em: 5/7/2007 


\section{Introdução}

Desde o início da existência da humanidade, há aproximadamente 200 milhões de anos, a amamentação tem sido a forma de garantia da sobrevivência da espécie devido aos nutrientes e fatores imunológicos transmitidos pelo leite materno ${ }^{(1)}$. Neste período, a composição do leite humano adequou-se às necessidades dos lactentes, estabelecendo perfeita relação nutricional e imunológica espécie-específica. Atualmente, são atribuídas vantagens nutricionais, imunológicas, cognitivas, psicoafetivas, econômicas e sociais à amamentação. Desta forma, a Organização Mundial de Saúde (OMS) recomenda o aleitamento materno até pelo menos dois anos, devendo ser praticado de forma exclusiva até o sexto mês de vida ${ }^{(2)}$. Entretanto, existem fatores que contribuem para a interrupção precoce da amamentação, dentre os quais o uso de medicamentos pela nutriz ${ }^{(3-5)}$.

Na prática, tem sido observada grande freqüência do uso de medicamentos durante a amamentação ${ }^{(6)}$. Deste modo, publicações sobre drogas e aleitamento materno são regularmente disponibilizadas pelo Ministério da Saúde ${ }^{(7)}$, pela Academia Americana de Pediatria (AAP) ${ }^{(8)}$, pela $\mathrm{OMS}^{(9)}$ e por Hale ${ }^{(10)}$, entre outros, no intuito de orientar os profissionais de saúde. Porém, face à dinâmica do conhecimento e do surgimento de novas drogas, as informações necessitam ser atualizadas constantemente para proporcionar maior segurança para o médico clínico, obstetra e pediatra, que, em geral, prestam assistência à mulher lactante. Com o avanço do conhecimento científico e da terapêutica, novos medicamentos surgem a cada dia no mercado. Novos fármacos são, portanto, prescritos também para mulheres em amamentação, aumentando o risco de desmame por desconhecimento do clínico a respeito da segurança do seu emprego na nutriz.

A associação entre uso de medicamentos pela nutriz e desmame ${ }^{(4,8)}$ reforça a importância de novas informações sobre o tema. Assim, o presente artigo é uma revisão e uma atualização bibliográfica sobre o uso de medicamentos durante o período de amamentação, visando contribuir com informações úteis para profissionais de saúde na assistência materno-infantil.

\section{Métodos}

Foram selecionados artigos nos bancos de dados eletrônicos Pubmed, Medline, Lilacs e SciELO nos últimos dez anos, nas línguas portuguesa, inglesa e espanhola, utilizando os descritores "aleitamento materno", "lactação", "leite humano" e "uso de medicamentos". Também foram utilizados livros, textos recentes e artigos considerados relevantes para a realização dessa revisão.

\section{Classificação dos fármacos para uso durante a amamentação}

Segundo Hale ${ }^{(10)}$, os medicamentos são classificados em categorias de risco para uso na lactação. Os de nível 1 (L1) são os mais seguros, enquanto que os de nível 5 (L5) são contra-indicados. Os níveis 2 (L2), 3 (L3) e 4 (L4) são considerados, respectivamente, seguros, moderadamente seguros e possivelmente perigosos. Para fins práticos, os fármacos de níveis 1 e 2 são apresentados conjuntamente na Tabela $1 \mathrm{~A}$ e 1B e os demais nas Tabelas 2, 3 e 4.

\section{Farmacologia e lactação}

Se necessário prescrever medicamentos durante a amamentação, é imprescindível o conhecimento dos fatores que determinam a segurança para uso nesse período. Tais fatores podem estar relacionados com os aspectos metabólicos e fisiológicos do leite humano, com a mulher, com o lactente ou com o fármaco. A composição do leite materno varia conforme a fase da lactação (colostro versus leite maduro) ou até mesmo durante uma mamada (leite anterior versus leite posterior). Tais alterações influenciam na extensão da transferência de fármacos do plasma para o leite, causando variações nas concentrações dos mesmos no leite materno ${ }^{(11)}$. Os fármacos transferem-se mais facilmente para o leite materno durante os primeiros dias de lactação (colostro), pois as células alveolares são menores e o espaço intercelular largo. A partir da segunda semana pós-parto, há a redução dos níveis de progesterona seguida de crescimento das células alveolares e estreitamento dos espaços intercelulares. Assim, ocorre redução da transferência de fármacos para o leite materno. Porém, a dose absoluta dos fármacos recebida pelo recém-nascido é baixa devido ao pequeno volume de colostro ingerido $(50 \text { a } 60 \mathrm{~mL} / \mathrm{dia})^{(5)}$.

Fatores que reduzem a capacidade da mãe de metabolizar ou excretar o fármaco podem aumentar a exposição do lactente ao mesmo ${ }^{(12)}$. Assim, cuidado especial deve ser tomado ao prescrever medicamentos para nutrizes com doenças hepáticas ou renais pelos níveis mais elevados e 
por maior tempo na circulação materna. A via pela qual o fármaco é administrado à mãe tem importância devido aos níveis alcançados no plasma materno e, posteriormente, no leite humano. Desta forma, muitos fármacos administrados topicamente ou inalados não atingem níveis plasmáticos significativos, possuindo níveis lácteos não mensuráveis. Muitos antibióticos, corticosteróides e retinóides, aplicados em áreas restritas, são pouco absorvidos por via transcutânea e praticamente indectáveis no plasma ${ }^{(5)}$.

A idade do lactente tem sido apontada como uma das mais importantes variáveis a ser considerada no momento de de- terminar a segurança do fármaco para uso durante a lactação. Estudo de revisão sobre efeitos adversos em lactentes de medicamentos utilizados pelas mães mostrou risco mais elevado de reações nos menores de dois meses (78\%). Apenas $4 \%$ das reações ocorreram em lactentes maiores de seis meses, período de maior maturidade metabólica hepática e de menor ingesta láctea devido à alimentação complementar ${ }^{(13)}$. Porém, Hale $e^{(5)}$ classifica o risco de efeitos adversos para o lactente segundo a idade como: baixo risco (seis a 18 meses), risco moderado (dois a seis meses) e alto risco (prematuros, recém-nascidos, lactentes clinicamente instáveis ou com função renal debilitada).

Tabela 1A - Fármacos seguros para uso durante a lactação

\begin{tabular}{|c|c|}
\hline Classes farmacológicas & Fármacos \\
\hline $\begin{array}{l}\text { Analgésicos e antiinflamatórios não } \\
\text { esteróides }\end{array}$ & $\begin{array}{l}\text { Acetominofen, azaprazone, celecoxib, cetorolaco, diclofenaco, fenoprofeno, } \\
\text { flurbiprofeno, ibuprofeno, piroxicam, rofecoxib }\end{array}$ \\
\hline Analgésicos opióides e antagonistas & $\begin{array}{l}\text { Alfentanil, buprenorfina, butorfanol, fentanil, meperidina", nalbufina, } \\
\text { naltrexona, propoxifeno }\end{array}$ \\
\hline Anestésicos e indutores anestésicos & Bupivacaína, halotano, lidocaína, propofol, ropivacaína \\
\hline Corticosteróides & $\begin{array}{l}\text { Beclometazona, budesonida, hidrocortisona, prednisolona, prednisona, } \\
\text { metilprednisolona }\end{array}$ \\
\hline Anti-histamínicos & $\begin{array}{l}\text { Cetirizina, desloratadina, difenidramina, dimenidrinato, loratadina, fexofenadina, } \\
\text { hidroxizine, levocabastina, olopatadina, prometazina, triprolidina }\end{array}$ \\
\hline Antitussígenos e mucolíticos & Dextrometorfano, guaifenesina \\
\hline Descongestionantes nasais & Fenilpropanolamina \\
\hline Broncodilatadores & $\begin{array}{l}\text { Albuterol, brometo de ipratrópio, isoetarina, isoproterenol, levalbuterol, } \\
\text { pirbuterol, salmeterol, terbutalina }\end{array}$ \\
\hline Antiasmáticos & Cromoglicato sódico, nedocromil \\
\hline Anti-hipertensivos & $\begin{array}{l}\text { Benazepril, captopril, enalapril, hidralazina, labetalol, metildopa, mepindolol, } \\
\text { minoxidil, nicardipina, nifedipina, nimodipina, nitrendipina, propranolol, } \\
\text { quinapril, timolol, verapamil }\end{array}$ \\
\hline Diuréticos & Acetazolamida, espironolactona, hidroclorotiazida \\
\hline Hipolipemiantes & Colesevelan, colestiramina \\
\hline Antiarrítmicos & Digoxina, disopiramida, mexiletine, quinidina, propafenona \\
\hline Aminas vasoativas & Adrenalina, dipivefrin, dobutamina, dopamina \\
\hline Antiácidos & $\begin{array}{l}\text { Cimetidina, esomeprazol, famotidina, hidróxido de magnésio, nizaditina, } \\
\text { omeprazol, pantoprazol, ranitidina, sucralfato }\end{array}$ \\
\hline Antieméticos e gastrocinéticos & Cisaprida, dimenidrinato, domperidona, metoclopramida, ondansetron \\
\hline Antidiarréicos & Kaolim, loperamida, pectina \\
\hline Laxante & Bisacodil, docusato, laxantes salinos e osmóticos \\
\hline
\end{tabular}

"uso no pós-parto imediato 
Tabela 1B - Fármacos seguros para uso durante a lactação

\begin{tabular}{|c|c|}
\hline Classes farmacológicas & Fármacos \\
\hline Antiparasitários & Permetrina, praziquantel \\
\hline Antibióticos & $\begin{array}{l}\text { Amicacina, amoxicilina, ampicilina, clavulanato de potássio, azitromicina, aztreonam, } \\
\text { carbenicilina, cefalosporinas }{ }^{*} \text {, claritromicina, clindamicina, clotrimazol, cloxacilina, } \\
\text { dicloxacilina, eritromicina, ertapenem, etambutol, floxacilina, furazolidona, gentamicina, } \\
\text { imipenem, kanamicina, lincomicina, loracarbef, metronidazol, minociclina, moxifloxacin, } \\
\text { mupirocina, nafcilin, nitrofurantoína, ofloxacin, penicilina G, piperacilina, polimixina B, } \\
\text { rifampicina, sulfisoxazol, ticarcilina, tetraciclina, trimetoprim, vancomicina }\end{array}$ \\
\hline Antivirais & Aciclovir, fanciclovir, lamivudina, valaciclovir \\
\hline Antifúngicos & $\begin{array}{l}\text { Cetoconazol, clotrimazol, fluconazol, griseofulvina, itraconazol, miconazol, nistatina, } \\
\text { terbinafina, tinidazol }\end{array}$ \\
\hline Antimaláricos & Hidroxicloroquina, mefloquine, quinina \\
\hline Antidiabéticos & Insulina, gliburida, metformin, miglitol \\
\hline Antitireoideanos & Propiltiouracil \\
\hline Anovulatórios & Etonogestrel, levonorgestrel, medroxiprogesterona, noretinodrel, noretindrona \\
\hline Hormônios & Levotiroxina, liotironina, desmopressina, tirotropina \\
\hline Anticoagulantes & Dalteparina, heparina, warfarin \\
\hline Hipnóticos e ansiolíticos & Nitrazepam**, zalepton, zopiclone \\
\hline Neurolépticos & Haloperidol, quetiapina, sulpiride \\
\hline Antidepressivos & $\begin{array}{l}\text { Amitriptilina, amoxapine, citalopram, clomipramina, desipramina, dotiepin, fluoxetina }{ }^{* * *} \text {, } \\
\text { fluvoxamina, imipramina, mirtazapina, paroxetina, nortriptilina, sertralina, trazodone }\end{array}$ \\
\hline Anticonvulsivantes & Ácido valpróico, carbamazepina, fenitoína, fosfenitoína, sulfato de magnésio \\
\hline Antienxaqueca & Eletriptan \\
\hline Relaxantes musculares & Baclofeno, mivacúrio \\
\hline Uterotônicos & Metilergonovina $^{* *}$, oxitocina \\
\hline Adoçantes & Aspartame \\
\hline Contraste e radioisótopos & Bário, C-racloripe, gadopentetato, ioxenol, metrizamida, metrizoato \\
\hline Antineoplásicos & Hidroxiuréia \\
\hline Vitaminas & Vitaminas $\mathrm{C}, \mathrm{D}, \mathrm{E}, \mathrm{K}$, piridoxina \\
\hline Sais minerais & Sais ferrosos, sais de zinco, flúor \\
\hline Ervas & Erva de São João (Hypercurium perforatum) \\
\hline Vacinas & $\begin{array}{l}\text { Doença de Lyme, DPT, febre tifóide, hepatite A, hepatite B, influenza, meningocócica C } \\
\text { conjugada, MMR, pólio oral, rubéola, varicela }\end{array}$ \\
\hline Imunoglobulinas & Hepatite B, RHO \\
\hline Outros & $\begin{array}{l}\text { Alopurinol, cafeína, carbamida, clorexedine, dornase, fator de coagulação VIla, filgastrin, } \\
\text { hilan G-F 20, infliximab, interferon alfa N3, injeções para alergia, lisina, nicotina adesivo/ } \\
\text { goma/spray, pamidronato, pentosan, pentoxifilina, pimecrolimus, piridostigmina, teste } \\
\text { tuberculínico (PPD) }\end{array}$ \\
\hline
\end{tabular}

*exceto cefditoren; **períodos curtos; ${ }^{* * *}$ após um mês de vida 
Tabela 2A - Fármacos moderadamente seguros para uso durante a lactação

\begin{tabular}{|c|c|}
\hline Classes farmacológicas & Fármacos \\
\hline $\begin{array}{l}\text { Analgésicos e antiinflamatórios } \\
\text { não esteróides }\end{array}$ & $\begin{array}{l}\text { AAS, diflunisal, etodolaco, indometacina, meloxican, mesalamina, nabumetona, } \\
\text { naproxeno*, olsalazina, oxaprozin, tolmetin }\end{array}$ \\
\hline $\begin{array}{l}\text { Analgésicos opióides } \\
\text { e antagonistas }\end{array}$ & $\begin{array}{l}\text { Codeína, hidromorfone, hidrocodone, meperidina**, metadona, morfina, } \\
\text { naloxona, oxicodone, pentazocina, tramadol }\end{array}$ \\
\hline $\begin{array}{l}\text { Anestésicos e indutores } \\
\text { anestésicos }\end{array}$ & $\begin{array}{l}\text { Articaína, dibucaína, mepivacaína, metohexital, óxido nitroso, procaína, } \\
\text { remifentanil, sevoflurano, tiopental }\end{array}$ \\
\hline Antiespasmóticos & Escopolamina, flavoxate, hiosciamina, metescolpolamina, oxibutinina \\
\hline Corticosteróides & $\begin{array}{l}\text { Betametasona, dexametasona, fludocortisona, flunisolida, fluticasona, } \\
\text { mometasona, prednicarbato, triancinolona }\end{array}$ \\
\hline Anti-histamínicos & Azelastina, trimeprazine \\
\hline Antitussígenos & Benzonatato \\
\hline Descongestionantes nasais & Fenilefrina, pseudoefedrina* \\
\hline Broncodilatadores & Difilina, formoterol, teofilina \\
\hline Anti-hipertensivos & $\begin{array}{l}\text { Acebutolol, amlodipina, atenolol, betaxolol, bisoprolol, candesartan, carteolol, } \\
\text { carvedilol, esmolol, fosinopril, guanfacine, isradipina, levobunolol, lisinopril, } \\
\text { metoprolol, nisoldipina, olmesartam, ramipril, sotalol, telmisartan }\end{array}$ \\
\hline Diuréticos & Ácido etacrínico, furosemida, indapamida, manitol, torsemide, triantereno \\
\hline Vasodilatadores & Isossorbida \\
\hline Antilipêmico & Ezetimibe, fluvastina, pravastatina \\
\hline Antiarrítmicos & Digitoxina, diltiazem, encainide, flecainide, procainamida \\
\hline Laxantes & Senna, cáscara sagrada, óleo de castor, polietilenoglicol \\
\hline Antidiarréicos & Difenoxilato, paregórico \\
\hline Antiácidos & Lansoprazol, rabeprazol \\
\hline Antieméticos & Dolasetron, granisetron, meclizine, proclorperazina, tropisetron \\
\hline Hipolipemiantes & Atorvastatina, fenofibrato, genfibrosil, sinvastatina \\
\hline Trombolíticos & Alteplase \\
\hline $\begin{array}{l}\text { Anticoagulantes e antiagregantes } \\
\text { plaquetários }\end{array}$ & Dipiridamol, enoxaparin, epoprostenol, lepirudin, tinzaparina \\
\hline Antiparasitários & Albendazol, ivermectina, mebendazol, pamoato de pirantel, tiabendazol \\
\hline Antibióticos & $\begin{array}{l}\text { Cefditoren, dalfopristina, daptomicina, diritromicina, doxiciclina, enoxacin, } \\
\text { fosfomicina, gatifloxacin, isoniazida, levofloxacin, linezolida, lomefloxacin, } \\
\text { meropenem, oxacilina, netilmicina, norfloxacin, pirazinamida, podofilox, } \\
\text { quinupristina, rifaximin, streptomicina, sulfadizina de prata, sulfametoxazol+trim } \\
\text { etoprim, sulfassalazina, telitromicina, tobramicina }\end{array}$ \\
\hline Antivirais & $\begin{array}{l}\text { Interferon beta } 1 \mathrm{~A} \text { e } 1 \mathrm{~B} \text {, nevirapina, oseltamivir, penciclovir, valganciclovir, } \\
\text { zanamivir, zidovudina }\end{array}$ \\
\hline Antifúngicos & Anfotericina $\mathrm{B}$, caspofungin, sulconazol, terconazol \\
\hline
\end{tabular}

"uso por períodos curtos; " uso no pós-parto imediato 
Tabela 2B - Fármacos moderadamente seguros para uso durante a lactação

\begin{tabular}{|c|c|}
\hline Classes farmacológicas & Fármacos \\
\hline Antimaláricos & Atovaquone, proguanil, primaquina \\
\hline Antidiabéticos & Acarbose, acetohexamida, exenatide, pioglitazona, pramlintide, rosiglitazona, glipizida \\
\hline Anovulatórios & Etinilestradiol, etonogestrel, drosperinona, levonorgestrel ${ }^{\star * *}$ \\
\hline Antitireoideano & Carbimazol, metimazol \\
\hline $\begin{array}{l}\text { Hormônios e inibidores } \\
\text { hormonais }\end{array}$ & $\begin{array}{l}\text { Corticotropina, estrogênio, estradiol, gonadorelin, gonadotrofina coriônica, ganirelix, } \\
\text { goserelin implante, FSH, melatonina, menotropina, mifepristona, octreotide, progesterona, } \\
\text { somatropina, triptolerin, vasopressina }\end{array}$ \\
\hline Hipnóticos e ansiolíticos & $\begin{array}{l}\text { Alprazolam, buspirona, butabarbital, butalbital, diazepam, droperidol, estazolam, } \\
\text { eszopiclone, flunitrazepam, flurazepam, halazepam, hidrato de cloral, lorazepam, } \\
\text { meprobamato, midazolam, oxazepam, pentobarbital, prazepam, secobarbital, temazepam, } \\
\text { triazolam, zolpidem }\end{array}$ \\
\hline Neurolépticos & Aripiprazol, flufenazina, perfenazina, risperidona \\
\hline Antidepressivos & $\begin{array}{l}\text { Amitriptilina, bupropiona, duloxetina, fluoxetina }{ }^{* * *} \text {, maprotilina, mirtazapina, moclobenida, } \\
\text { venlafaxina }\end{array}$ \\
\hline Antiepiléticos & $\begin{array}{l}\text { Fenobarbital, gabapentina, lamotrigina, levertiracetam, oxcarbazepina, primidona, tiagabina, } \\
\text { topiramato, vigabatrina, zolmitriptam }\end{array}$ \\
\hline Antienxaquecosos & Almotriptam, frovatriptam, isometepteno, naratriptam, rizatriptam, sumatriptam \\
\hline Relaxantes musculares & Carisoprodol, metaxalona, metocarbamol, orfenadrina, toxina botulínica \\
\hline Uterotônicos & Carboprost trometamina, ergonovina \\
\hline Imunoglobulinas & Adalimumab, alemtuzumab \\
\hline Antipsoriático & Antralina, tazaroteno \\
\hline Imunossupressores & Azatioprina, mercaptopurina, metotrexate ${ }^{*}$ \\
\hline Contrastes e radioisótopos & $\begin{array}{l}\text { Gadodiamina, gadoteridol, gadoversetamina, índio 111, iopamidol, ioversol 160-350, } \\
\text { mangafodipir }\end{array}$ \\
\hline Vitaminas e análogos & Vitamina A, ácido nicotínico (B3), calcipotriene, calcitriol, doxercalciferol, tretinoína \\
\hline Minerais & Cromo, selênio \\
\hline Ervas e fitoterápicos & $\begin{array}{l}\text { Alho, calêndula, camomila germânica, equinácea, fenogreco, ginko biloba, ginseng, milk thistle } \\
\text { (silybum marianum), óleo de mameluca, óleo de prímula da noite, valeriane officinalis }\end{array}$ \\
\hline Vacinas & Antrax, cólera, febre amarela, hemófilus influenza B, raiva, toxóide diftérico, toxóide tetânico \\
\hline Outros & $\begin{array}{l}\text { Ácido azelaico, adapalene, alendronato, alosetron, amantadina, anakinra, arginina, atropina, } \\
\text { balsalazide, benzotropina, bismuto, brimonidina, calcitonina, capsaicin, carbidopa, condroitina, } \\
\text { darifenacin, deferoxamina, dimetilsulfoxide, dinoprostone, donepezil, epoetin alfa, etanercept, } \\
\text { etanol, etidronato, fenazopiridina, fludeoxiglicose, fluocinolona+hidroquinona+tretinoína, } \\
\text { fluoresceína, glatiramer, glicopirrolato, glucosamina, hidroquinona, histamina, latanoprost, } \\
\text { metacolina, metilbenzamida }{ }^{* \star \star x \star} \text {, metilfenidato, midodrine, misoprostol, montelukast, natalizumab, } \\
\text { pilocarpina, risedronato, ritodrina, sacarina, sildenafil, sincalide, succimer, tacrolimus, tálio-201, } \\
\text { tegaserod, tolteronid, ursodiol, violeta genciana, xenon-133, zafirlukast }\end{array}$ \\
\hline
\end{tabular}

"uso por períodos curtos; " uso durante período neonatal; "**tuso regular ou contracepção de emergência; ${ }^{* * * *}$ uso como repelente 
Tabela 3 - Fármacos potencialmente perigosos durante a lactação

\begin{tabular}{|c|c|}
\hline Classes farmacológicas & Fármacos \\
\hline $\begin{array}{l}\text { Analgésicos e antiinflamatórios } \\
\text { não esteróides }\end{array}$ & Colchicina, naproxeno* \\
\hline Anti-histamínicos & Clemastina, doxilamina, tripelenamina \\
\hline Mucolíticos & lodeto de potássio \\
\hline Descongestionantes nasais & Efedrina, pseudoefedrina** \\
\hline Anti-hipertensivos & $\begin{array}{l}\text { Bepridil, doxazosin, flunarizina, fosinopril*, nadolol, prazosin, quinapril }{ }^{*} \text {, reserpina, } \\
\text { telmisartan*, terazosin, valsartan }\end{array}$ \\
\hline Vasodilatadores & Nitroglicerina, nitroprussiato, nitritos e nitratos \\
\hline Diuréticos & Bendroflumetiazida \\
\hline Antianginosos & Nitratos, nitritos e nitroglicerina \\
\hline Antiarrítmicos & Tocainide \\
\hline $\begin{array}{l}\text { Anticoagulantes e } \\
\text { antiagregantes plaquetários }\end{array}$ & Argatroban, ticlopidina \\
\hline Antieméticos e antivertiginosos & Trimetobenzamida \\
\hline Escabicida e pediculicida & Lindano \\
\hline Antimaláricos & Pirimetamina, quinacrina \\
\hline Antibióticos & Ácido nalidíxico, cloranfenicol, dapsona, grepafloxacin, trovafloxacin \\
\hline Antivirais & Foscarnet, ribavirina \\
\hline Imunossupressores & Cisplatina, leflunomide, micofenolato, sirulimus \\
\hline Antidiabéticos & Glimepirida, repaglinida \\
\hline Antiprolactinogênicos & Cabergoline \\
\hline Anorexígenos & Dexfenfluramina, dextroanfetamina, fentermina, sibutramina \\
\hline Uterotônicos & Metilergonovina* \\
\hline Relaxantes musculares & Dantrolene, tizanidina \\
\hline Compostos radioativos & Cobre 64, gálio 67, índio 111, iodo 123 e 125, iodo 131, tecnécio 99 \\
\hline Neurolépticos & Loxapine, mesoridazina, pimozide, ziprasidona \\
\hline Antidepressivos & Lítio, nefazodone, tioridazida, tiotixeno \\
\hline Antiparkinsonianos & Levodopa, pramipexazol, ropirinol \\
\hline Antiepiléticos & Etossuximida, felbamato \\
\hline Antimigrânea & Ergotamina \\
\hline Antivertiginosos & Flunarizina \\
\hline Vitaminas & Piridoxina ${ }^{* * *}$ \\
\hline Ervas & Cohosh preto, funcho (Foeniculum vulgare), sálvia (salvia officinalis) \\
\hline Vacina & Varicela \\
\hline Outros & $\begin{array}{l}\text { Atomoxetine, betanecol, diclomina, dorzolamida, doxepin creme, éter, formaldeído, } \\
\text { hexaclorofeno, iodo-povidine, leflunomide, letrozol, modafinil, penicilamina, solifenacin, } \\
\text { pimecrolinus }{ }^{*+*+*} \text {, metilergonovina*, trastrumuzab }\end{array}$ \\
\hline
\end{tabular}

"uso no período neonatal; "uso crônico; ***uso em altas doses; ;*** uso no mamilo 
A relação entre idade do lactente e risco de efeito adverso sofre influência do tipo de aleitamento praticado, se exclusivo ou não, e do grau de maturidade dos principais sistemas de eliminação de fármacos. Além disso, a barreira hematoencefálica é imatura em recém-nascidos e lactentes jovens, havendo aumento da passagem de fármacos lipossolúveis que atuam no sistema nervoso central ${ }^{(14)}$.

Em relação aos fármacos, a transferência para o leite materno depende das seguintes características:

1) peso molecular;

2) lipossolubilidade;

3) capacidade de ligação às proteínas;

4) grau de ionização;

5) meia-vida de eliminação;

6) biodisponibilidade;

7) concentração plasmática materna.

A passagem do fármaco entre o plasma e o leite é bidirecional. Quando a concentração do fármaco livre, não ionizado, no leite é mais elevada que a existente no plasma, ocorre a transferência deste fármaco para o plasma. Assim, ordenhar e descartar o leite não acelera de forma efetiva a eliminação da maioria dos fármacos excretados pelo leite ${ }^{(15)}$. Desta forma, o conhecimento farmacológico pode auxiliar o profissional no momento da prescrição, devendo-se optar por fármacos com baixa transferência do plasma para o leite (Tabela 5)

\section{Métodos de determinação da taxa de transferência do fármaco para o leite materno}

A exposição do lactente ao fármaco pode ser estimada quantitativamente por meio de algumas medidas. As mais utilizadas são a razão leite-plasma e a dose relativa no lactente. Essas medidas tornam-se mais importantes quando as mulheres fazem uso de fármacos por longos períodos ${ }^{(18)}$ ou quando o fármaco em questão apresenta risco de dano à saúde da criança, mesmo após única exposição.

A razão leite-plasma foi muito usada para estimar a quantidade do fármaco transferido para o leite. Ela consiste na

Tabela 4 - Fármacos contra-indicados para uso durante a lactação

\begin{tabular}{ll}
\hline Classes farmacológicas & Fármacos \\
\hline Analgésicos e antiinflamatórios & Antipirina \\
Antiarrítmico & Amiodarona \\
Anorexígenos & Dietilpropiona \\
Hormônios e antagonistas hormonais & Danazol, dietilestilbestrol, leuprolide, tamoxifeno \\
Metais pesados & Chumbo, mercúrio \\
Compostos radioativos & Estrôncio-89 \\
Hipnóticos & Brometos \\
Antiparkinsonianos & Bromocriptina \\
Antidepressivos & Doxepin \\
Antiepiléptico & Zonisamida \\
Antineoplásicos & Busulvan, ciclofosfamida, citarabina, clorambucil, doxorubicina, \\
& fluoruracil, metotrexate*, mitoxantrone, paclitaxel \\
Antipsoriático & Etretinato \\
Antiartrítico & Sais de ouro \\
Drogas de abuso & Ácido gama hidroxibutírico, maconha, cocaína, fenciclidina, heroína, LSD \\
Ervas & Borage, chá de kombucha, cohosh azul, confrei, kava-kava \\
Outros & Dissulfiram, isotretinoína \\
\hline
\end{tabular}

"uso crônico 
razão entre concentrações do fármaco no plasma e no leite em estado de equilíbrio:

razão leite-plasma=concentração do fármaco no leite/ concentração do fármaco no plasma

Assim, uma razão leite-plasma igual a 4 significa que a concentração da medicação no leite é quatro vezes maior que a concentração plasmática ${ }^{(19)}$. Mas esta medida tem pouco valor prático, pois não leva em consideração o potencial tóxico do fármaco. Razão maior que 1 não traz preocupação quando a concentração do fármaco no plasma materno é muito baixa ou se o fármaco não é absorvido pelo lactente ${ }^{(4)}$. Embora as concentrações de muitos fármacos no plasma e no leite sejam flutuantes, são utilizadas medidas fixas para o cálculo da razão ${ }^{(11)}$.

A dose relativa no lactente é uma estimativa da percentagem da dose materna recebida pelo lactente por meio do leite, utilizando o seguinte cálculo:

dose relativa do lactente $(\%)=$ dose absoluta no lactente $(\mu \mathrm{g} / \mathrm{kg} / \mathrm{dia}) / \mathrm{dose}$ materna $(\mu \mathrm{g} / \mathrm{kg} / \mathrm{dia}) \times 100$

Esse valor é expresso em percentagem da dose materna e corresponde à proporção da dose materna recebida pelo lactente ${ }^{(20)}$. Usualmente, a dose relativa do lactente deve ser menor que $10 \%$ para que o fármaco seja considerado seguro ${ }^{(4)}$. O fármaco é considerado de risco elevado para efeitos adversos em lactentes quando este valor supera $25 \%{ }^{(15)}$. Porém, o método também possui limitações para aplicação prática, pois se baseia no princípio de que mãe e filho possuem mesma absorção, metabolização e excreção ${ }^{(12)}$. Desta forma, não existe um método confiável e eficaz para avaliar a taxa de transferência do fármaco. Outros fatores, portanto, devem ser considerados, como potencial tóxico do fármaco, dose, duração do tratamento, idade do lactente, volume de leite consumido, segurança do fármaco para o lactente, biodisponibilidade do fármaco tanto para mãe quanto para o lactente e risco de redução da lactogênese. Portanto, pela avaliação cuidadosa da real necessidade do uso de medicamentos bem como também da escolha da medicação, a amamentação poderá ser mantida na grande maioria das mães em uso de medicamentos.

\section{Fármacos que podem alterar o volume do leite materno}

Existem medicamentos que podem alterar o volume do leite materno, aumentando ou diminuindo sua produção. Denomina-se "galactagogo" o fármaco que possui efeito potencial de aumentar o volume de leite pela nutriz. Esses

Tabela 5 - Características farmacológicas que influenciam a transferência de fármacos para o leite materno

\begin{tabular}{ll}
\hline Características do fármaco & Mecanismos \\
\hline Peso molecular & Fármacos com baixo peso molecular atingem mais facilmente o leite materno ${ }^{(16)}$. \\
& Moléculas com pesos moleculares menores que 200 kilodaltons atravessam melhor \\
& os poros das membranas. Moléculas pequenas como uréia e etanol atravessam o \\
capilar endotelial materno e a célula alveolar por difusão passiva & ${ }^{(12)}$.
\end{tabular}

Lipossolubilidade

Ligação a proteínas

Grau de ionização

Meia-vida

Biodisponibilidade

Concentração plasmática materna
Fármacos lipossolúveis atingem rapidamente o compartimento lácteo, pois atravessam mais facilmente a barreira celular lipoprotéica. Há maior concentração no leite maduro devido à maior ligação com lipídes ${ }^{(5)}$; exemplos: sulfonamidas e cloranfenicol.

Fármacos com baixa afinidade por proteínas plasmáticas podem ser excretados com mais facilidade no leite ${ }^{(16)}$. Exemplo: diazepam.

O leite humano é um pouco mais ácido $(\mathrm{pH}=7,1)$ do que o plasma $(\mathrm{pH}=7,4)$. Fármacos que são bases fracas tendem a formar íons no leite, favorecendo maior concentração(17). Exemplo: betabloqueadores.

Fármacos com elevada meia-vida de eliminação mantêm níveis circulantes por maior tempo no sangue materno e, por conseguinte, no leite materno ${ }^{(5)}$. Ficar atento aos fármacos que possuem metabólitos ativos; por exemplo: preferir lorazepam em vez de diazepam.

Fármacos com baixa biodisponibilidade e pouco absorvidos pelo lactente são ideais para uso na lactação(15). Exemplos: heparina, aminoglicosídeos e insulina são seguros para uso pela nutriz.

A difusão passiva dos fármacos ocorre por gradiente de concentração. Elevados níveis séricos tendem a produzir elevados níveis do fármaco no leite ${ }^{(17)}$. 
fármacos atuam como bloqueadores de dopamina na hipófise. Entretanto, não há evidências de que tais medicamentos estimulem a produção láctea em mulheres com níveis elevados de prolactina ou com tecido mamário inadequado à lactação. Contudo, em mães de crianças pré-termo, tais medicamentos podem ser eficazes ${ }^{(20)}$. Apesar de numerosos fármacos apresentarem efeito potencial para aumentar o volume de leite ou induzir a lactação (Tabela 6), somente a metoclopramida e a domperidona são utilizadas algumas vezes na prática clínica ${ }^{(21)}$. Entretanto, deve-se estar atento para a ocorrência de reações adversas tanto na mãe quanto no lactente, como reações extrapiramidais ou distônicas. Cabe ressaltar que os estímulos mais valiosos para a produção e manutenção do volume de leite materno são a sucção do complexo aréolomamilar pelo lactente e a ordenha das mamas ${ }^{(18)}$.

Vários são os fármacos com relato de supressão da produção láctea. A maioria deles age como agonista dopaminérgico, suprimindo a liberação de prolactina. A Tabela 6 mostra os fármacos desse grupo. $\mathrm{O}$ uso de qualquer um desses fármacos pode representar risco potencial de deficit ponderal, principalmente durante o puerpério imediato, período mais sensível para a supressão da lactação. Deve-se, portanto, retardar ao máximo a sua introdução. Na vigência do uso desses fármacos, o crescimento do lactente deve ser rigorosamente acompanhado.

\section{Princípios básicos para uso de fármacos durante a amamentação}

Os estudos que procuram determinar a segurança dos medicamentos para uso durante a amamentação são realizados com pequenas séries de casos ou relatos de casos individuais. Assim, o princípio fundamental da prescrição de medicamentos para mães lactantes baseia-se, sobretudo, no risco versus benefício ${ }^{(18)}$. Os aspectos a serem avaliados incluem os benefícios da amamentação, o impacto dos sintomas e da doença sobre a saúde materna, além do desejo materno ${ }^{(23)}$. $\mathrm{Na}$ Tabela 7 são mencionados alguns aspectos práticos para a tomada de decisões pelo profissional de saúde, adaptados e modificados das normas básicas para prescrição de fármacos às mães durante a lactação, considerados na última revisão sobre o tema pela $\mathrm{AAP}^{(8)}$.

\section{Uso de medicamentos pela nutriz e desmame}

O uso de medicamentos pela mulher durante a amamentação é uma prática muito freqüente, como mostrado em vários estudos em diferentes fases da lactação ${ }^{(25)}$. No período intraparto, um estudo multicêntrico demonstrou uso de medicamentos por quase $80 \%$ das mulheres ${ }^{(26)}$. Passmore et al ${ }^{(27)}$ encontraram uso de medicamentos no pós-parto imediato por $99 \%$ da puérperas. Na Noruega, Matheson ${ }^{(3)}$ observou o emprego de pelo menos um fármaco por $25 \%$ das nutrizes com bebês entre três e cinco meses. Na Dinamarca, estudo realizado com 16.001 mulheres mostrou que 34\% delas receberam prescrição medicamentosa nos primeiros três meses do pós-parto: $43 \%$ correspondiam a fármacos seguros, $4,8 \%$ eram possivelmente perigosos e $35,8 \%$ não estavam classificados pelo sistema sueco ${ }^{(28)}$. Hale ${ }^{(6)}$, citando Bennett, descreve o uso de medicamentos na primeira semana pós-parto por 90 a $99 \%$ das nutrizes. Em maternidades de Belo Horizonte, Minas Gerais, foi observada a prescrição de fármacos em 96,2\% das mulheres no pós-parto imediato ${ }^{(29)}$. Em Itaúna, município próximo à capital mineira, 100\% das puérperas receberam medicamentos no pós-parto imediato, com média de 4,3 fármacos por mulher. Após a alta hospitalar, $98 \%$ das mulheres relataram o emprego de pelo menos um fármaco ${ }^{(30)}$.

A freqüente necessidade de medicamentos na lactação deve ser encarada com preocupação devido à conhecida relação entre tal uso e o desmame. Ito $e t a^{(4)}$ demonstraram que mulheres em tratamento farmacológico de doenças crônicas - epilepsia, hipertireoidismo, doenças inflamatórias intestinais - iniciavam a amamentação menos freqüentemente do que as mulheres da população geral e, quando iniciavam, desmamavam seus filhos mais precocemente.

Tabela 6 - Fármacos que podem alterar o volume de leite materno

\section{Efeito sobre o volume de leite Fármacos}

$\begin{array}{ll}\text { Aumento } & \text { Domperidona, metoclopramida, sulpiride, clorpromazina, hormônio de crescimento, } \\ \text { hormônio secretor de tireotropina, fenogreco }\end{array}$

Redução

Estrógenos, bromocriptina, cabergolide, ergotamina, ergometrina, lisurida, levodopa,
pseudoefedrina, álcool, nicotina, brupropiona, diuréticos, testosterona

Fonte: Lamounier et al, 2007(22) 
Em Montes Claros, Minas Gerais, a análise de 50 mulheres com interrupção do aleitamento demonstrou que, em $20 \%$, esta se relacionou ao uso de medicamentos ${ }^{(31)}$. Em Itaúna, um estudo com 155 mulheres que desmamaram seus filhos, mostrou que $4,5 \%$ delas atribuíram tal fato ao emprego de medicamentos durante a lactação ${ }^{(30)}$.

A interrupção da amamentação durante o uso de medicamentos só deveria se justificar quando o fármaco em questão fosse contra-indicado neste período. Porém, na literatura, são descritos outros fatores utilizados como justificativa para a prática do desmame na vigência do uso de medicamento pela nutriz, dentre eles: o desconhecimento dos profissionais de saúde sobre o tema, as informações não científicas em bulas de medicamentos, a escassez de informações na literatura sobre a segurança dos fármacos na lactação e, enfim, o receio materno de usar medicamentos neste período ${ }^{(18)}$. Deste modo, o desconhecimento dos profissionais de saúde sobre o tema é citado como fator relacionado ao desma$m e^{(8,13,29)}$. Freqüientemente, médicos aconselham lactantes em uso de medicamentos a suspender a amamentação, sem avaliar as possíveis consequiências não só para o lactente, mas também para o suprimento lácteo materno ${ }^{(5)}$. No estudo de Chaves $^{(30)}$, a interrupção do aleitamento materno em sete mulheres ocorreu pelo uso de medicamento, seis das quais por indicação médica. Porém, em entrevistas com as nutrizes, nenhum medicamento era contra-indicado na amamentação. Na Lituânia, observou-se que 21 a 23\% dos desmames estavam direta ou indiretamente relacionados ao emprego de medicamentos pelas mães. Os autores concluíram que, na maioria das vezes, a falta de informação

Tabela 7 - Princípios básicos para uso de fármacos durante a amamentação

\begin{tabular}{|c|c|}
\hline Princípios & Considerações \\
\hline $\begin{array}{l}\text { Risco versus benefício } \\
\text { do fármaco }\end{array}$ & $\begin{array}{l}\text { Avaliar a necessidade da terapia medicamentosa. Nesse caso, a consulta entre o pediatra } \\
\text { e o obstetra ou o clínico é muito útil. O fármaco prescrito deve ter um benefício reconhecido } \\
\text { para a condição na qual está sendo indicado. }\end{array}$ \\
\hline $\begin{array}{l}\text { Experiência com o } \\
\text { fármaco }\end{array}$ & $\begin{array}{l}\text { Preferir um fármaco já estudado, sabidamente seguro para a criança e pouco excretado no } \\
\text { leite humano. Exemplo: prescrever paracetamol em vez de ácido acetilsalicílico; penicilinas } \\
\text { em vez de cloranfenicol. }\end{array}$ \\
\hline Via de administração & Preferir a terapia tópica ou local à oral e à parenteral, quando possível e indicado \\
\hline Tempo de ação & $\begin{array}{l}\text { Evitar fármacos de ação prolongada pela maior dificuldade de excreção pelo lactente. Exemplo: } \\
\text { preferir midazolam ao diazepam. }\end{array}$ \\
\hline $\begin{array}{l}\text { Horário de } \\
\text { administração }\end{array}$ & $\begin{array}{l}\text { Programar o horário de administração do fármaco à mãe a fim de evitar que o pico do } \\
\text { medicamento no sangue e no leite materno coincida com o horário da amamentação. Em } \\
\text { geral, a exposição do lactente ao fármaco pode ser diminuída, prescrevendo-o para a mãe } \\
\text { imediatamente antes ou logo após a mamada. Outra opção é ministrar o medicamento antes } \\
\text { do maior período de sono da criança. }\end{array}$ \\
\hline Níveis séricos & $\begin{array}{l}\text { Escolher medicamentos que alcancem níveis mínimos no leite. Por exemplo, os antidepressivos } \\
\text { sertralina e paroxetina possuem níveis lácteos bem mais baixos que a fluoxetina. }\end{array}$ \\
\hline Dosagem sérica & $\begin{array}{l}\text { Quando possível, dosar o fármaco na corrente sangüínea do lactente se houver risco para a criança, } \\
\text { como nos tratamentos maternos prolongados, a exemplo do uso de anticonvulsivantes. }\end{array}$ \\
\hline $\begin{array}{l}\text { Evitar fármacos } \\
\text { combinados }\end{array}$ & $\begin{array}{l}\text { Preferir o uso de medicamentos com um só fármaco, evitando combinações. Exemplo: usar apenas } \\
\text { paracetamol em vez de apresentações contendo paracetamol, ácido acetilsalicílico e cafeína. }\end{array}$ \\
\hline Observar a criança & $\begin{array}{l}\text { Orientar a mãe para observar a criança com relação aos possíveis efeitos colaterais, tais como alteração } \\
\text { do padrão alimentar, hábitos de sono, agitação, tônus muscular, distúrbios gastrintestinais. }\end{array}$ \\
\hline $\begin{array}{l}\text { Coletar leite e, se } \\
\text { preciso interromper } \\
\text { aleitamento. }\end{array}$ & $\begin{array}{l}\text { Orientar a mãe para retirar o seu leite com antecedência e estocar em congelador para } \\
\text { alimentar o bebê no caso de interrupção temporária da amamentação. Sugerir também } \\
\text { ordenhas periódicas para manter a lactação. }\end{array}$ \\
\hline $\begin{array}{l}\text { Informar aos pais a } \\
\text { respeito do fármaco }\end{array}$ & $\begin{array}{l}\text { Os pais devem ser informados em relação à ausência de informações sobre fármaco prescrito } \\
\text { para uso durante a amamentação e sobre os riscos de possíveis efeitos colaterais sobre o } \\
\text { lactente, principalmente em medicamentos de uso crônico. Alguns autores recomendam obter } \\
\text { o termo de consentimento informado por escrito }\end{array}$ \\
\hline
\end{tabular}


leva médicos a orientarem equivocadamente o desmame na vigência do uso de medicamentos ${ }^{(32)}$.

$\mathrm{O}$ uso adequado de medicamentos durante a amamentação também pode ser influenciado de forma negativa pela informação contida em bulas. A indústria farmacêutica baseia-se quase sempre em questões legais, e não em razões clínicas, ao informar possíveis efeitos de medicamentos durante a amamentação ${ }^{(10)}$. Bulas de medicamentos sabidamente seguros durante a lactação contêm orientações que os contra-indicam durante esse período ${ }^{(13,22)}$. Além disto, constata-se também que muitas bulas são omissas quanto à utilização de fármacos pela mãe durante a lactação, dificultando a tomada de decisão pelo profissional de saúde sobre a prescrição ou a continuidade da amamentação. Em estudo sobre informações acerca da segurança dos antiinflamatórios não esteróides durante a lactação, Chaves et al ${ }^{(33)}$ encontraram $90 \%$ de discordância entre as informações contidas em bulas com as informações científicas sobre o tema.

Embora o conhecimento a respeito dos fármacos na lactação tenha sido muito ampliado, ainda não se conhecem os efeitos colaterais de muitos medicamentos utilizados pela nutriz para as crianças amamentadas ${ }^{(7)}$. A modificação da composição das fórmulas infantis, como a introdução dos ácidos graxos poliinsaturados de cadeia longa, só foi aprovada após numerosos estudos que comprovaram a sua segurança para o lactente. Assim, seria esperada a mesma preocupação quando a composição do leite materno é modificada pela introdução de fármacos ingeridos pela mãe. Contudo, os efeitos de muitos fármacos, novos ou não, ainda não foram devidamente estudados ou apresentam divergências na literatura, quando utilizados na lactação. $\mathrm{Na}$ última revisão da $\mathrm{AAP}^{(8)}$ sobre o tema foram incluídos 233 fármacos. Já a maior revisão acerca do assunto em questão inclui 774 fármacos ${ }^{(10)}$. Se considerarmos que, no Brasil, são comercializados aproximadamente 1.500 fármacos, faltam informações sobre aproximadamente $45 \%$ do total ${ }^{(22)}$. Os antiinflamatórios não hormonais estão entre os fármacos mais utilizados no mundo, inclusive pelas nutrizes. Porém, dentre os 27 antiinflamatórios desta classe comercializados no Brasil, foram encontradas referências sobre a segurança para uso durante a amamentação em apenas $14(52 \%)^{(33)}$.

Outro importante fator de risco para o desmame é o receio materno do possível prejuízo do medicamento ao lactente. Ito et $a^{(34)}$ realizaram estudo prospectivo com 203 nutrizes que receberam prescrição médica de antibióticos. Após consulta telefônica a serviço de informação sobre medicamentos durante a lactação, todas foram orientadas a usar o antibiótico prescrito e manter a amamentação, porém $15 \%$ não iniciaram o tratamento e $7 \%$ suspenderam a amamentação. Winans ${ }^{(35)}$, em estudo de revisão sobre o uso de antidepressivos durante a lactação, relata que muitas mulheres e médicos evitam o aleitamento durante o emprego desses medicamentos pela ausência de dados sobre sua segurança. Matheson et al ${ }^{(36)}$ observaram que mais da metade das nutrizes no pós-parto acreditava que o risco de uso de fármacos durante a lactação seria igual ou maior que o uso dos mesmos durante a gravidez.

\section{Conclusões}

A associação entre uso de medicamentos pela nutriz e desmame revela a necessidade de constante atualização dos profissionais que prescrevem ou orientam as nutrizes sobre a segurança de medicamentos durante a lactação. As vantagens e a importância do aleitamento materno são bem conhecidas. Assim, a amamentação somente deverá ser interrompida diante de evidência substancial de que o fármaco usado pela nutriz é nocivo para o lactente. À luz dos conhecimentos atuais, pouquíssimos fármacos contemplam tal critério. Contudo, devido aos riscos potenciais de efeitos colaterais ou supressão da produção láctea que alguns medicamentos possuem, deve-se fazer opção por aqueles já estudados, pouco excretados no leite materno ou que não tenham risco aparente para a saúde da criança.

A carência de informações sobre a segurança para uso na lactação de grande parte dos fármacos dificulta a tomada de decisão no momento da prescrição ou orientação pelo profissional de saúde. Faz-se necessário maior investimento em pesquisas que determinem o grau de segurança dos fármacos durante a lactação. $\mathrm{O}$ conhecimento farmacológico permite ao profissional propiciar um tratamento adequado à nutriz, além de contribuir para a manutenção do aleitamento materno. Uma avaliação cuidadosa da real necessidade de uso da medicação quase sempre permite compatibilizar o tratamento medicamentoso com a amamentação. 


\section{Referências bibliográficas}

1. Ribeiro R, Sigulem DM, Novo NF, Juliano $Y$, Cury MCFS. Atenção à saúde da criança: um enfoque histórico e evolutivo. Rev latros 2002;17:42-9.

2. World Health Organization [homepage na Internet]. Geneva: Report of the expert consultation on the optimal duration of exclusive breastfeeding, 2001 [citado em 22 de fevereiro de 2007]. Disponível em: http://whqlibdoc.who. int/hq/2001/WHO_NHD_01.09.pdf

3. Matheson I. Drugs taken by mothers in the puerperium. BMJ 1985;290:1588-9.

4. Ito S. Drug therapy for breastfeeding women. N Engl J Med 2000;343:118-26.

5. Hale TW. Drug therapy and breastfeeding: pharmacokinetics, risk factors, and effects on milk production. Neoreviews 2004;5:e164-72. Disponível em: http://neoreviews.aappublications.org/cgi/reprint/neoreviews;5/4/e164

6. Hale TW. Maternal medications during breastfeeding. Clin Obstet Gynecol 2004;47:696-711.

7. Ministério da Saúde. Secretaria de Políticas de Saúde. Área técnica de Saúde da Criança. Amamentação e uso de drogas. Brasília: Ministério da Saúde; 2000.

8. American Academy of Pediatrics. Committee on drugs. The transfer of drugs and other chemicals into human milk. Pediatrics 2001;108:776-89.

9. World Health Organization/Unicef. Breastfeeding and maternal medication. Recommedations for drugs in the eleventh $\mathrm{WHO}$ model list of essential drugs. [documento na Internet]. Genebra: WHO/Unicef; 2002 [citado em 2 de fevereiro de 2007]. Disponivel em: http://www.who.int/child-adolescent-ealth/NewPublications/NUTRITION/BF_Maternal_Medication.pdf

10. Hale TW. Medications and mothers' milk. $12^{\text {th }}$ ed. Amarillo: Pharmasoft Publ; 2006.

11. Begg EJ, Duffull SB, Hackett LP, llett KF. Studying drugs in human milk: time to unify the approach. J Hum Lact 2002;18:323-32.

12. Howard CR, Lawrence RA. Drugs and breastfeeding. Clin Perinatol 1999;26:447-78.

13. Anderson PO, Pochop LS, Manoguerra AS. Adverse drug reactions in breastfed infants: less than imagined. Clin Pediatr (Phila) 2003;42:325-40.

14. Rivera-Calimlin L. The significance of drugs in breast milk. Pharmacokinetic considerations. Clin Perinatol 1987;14:51-70.

15. Anderson PO. Amamentação e uso de drogas. In: Carvalho MR, Tamez RN. Amamentação: bases científicas. $2^{\mathrm{a}}$ ed. Rio de Janeiro: Guanabara Koogan; 2005. p. 246-66.

16. Auerbach KG. Breastfeeding and maternal medication use. J Obstet Gynecol Neonatal Nurs 1999;28:554-63.

17. Berlin CM, Briggs GG. Drugs and chemicals in human milk. Semin Fetal Neonatal Med 2005;10:149-59.

18. Chaves RG, Lamounier JA. Uso de medicamentos durante a lactação. J Pediatr (Rio J) 2004;80(Suppl):S189-98.

19. Marks JM, Spatz DL. Medications and lactation: what PNPs need to know. J Pediatr Health Care 2003;17:311-7.
20. Hale TW. Medications in breastfeeding mothers of preterm infants. Pediatr Ann 2003;32:337-47.

21. Gabay MP. Galatogogues: medications that induce lactation. J Hum Lact 2002;18:274-9.

22. Lamounier JA, Chaves RG, Vieira GO. Medicamentos e amamentação. In Lopes FA, Campos Jr D. Tratado de pediatria da sociedade brasileira de pediatria. São Paulo: Manole; 2007. p. 327-34.

23. Pheula GF, Dalgalarrondo P, Celeri EH, Vilela WA. Uso de psicofármacos na lactação: revisão e proposta de manejo. J Bras Psiquiatr 2003;52:413-25.

24. Wisner KL, Perel JM, Findling RL. Antidepressant treatment during breastfeeding. Am J Psychiatry 1996;153:1132-7.

25. Ostrea EM, Mantaring JB, Silvestre MA. Drugs that affect the fetus and newborn infant via the placenta or breast milk. Pediatr Clin North Am 2004 51:539-79.

26. Collaborative Group on Drug Use in Pregnancy. Medication during pregnancy: an intercontinental cooperative study. Int J Gynaecol Obstet 1992; 39:185-96.

27. Passmore CM, McElnay JC, D'Arcy PF. Drugs taken by mothers in the puerperium: inpatient survey in Northern Ireland. Br Med J 1984;289:1593-6.

28. Olesen C, Sørensen HT, de Jong-van den Berg L, Olsen J, Steffensen FH. Prescribing during pregnancy and lactation with reference to the Swedish classification system. A population-based study among Danish women. The Euromap Group. Acta Obstet Gynecol Scand 1999;78:686-92.

29. Lamounier JA, Cabral CM, Oliveira BC, Oliveira AB, Oliveira Jr AM, Silva APA. O uso de medicamentos em puérperas interfere nas recomendações ao aleitamento materno? J Pediatr (Rio J) 2002;78:57-61.

30. Chaves RG. Situação do aleitamento materno e do uso de medicamentos pela nutriz no primeiro ano de vida da criança em Itaúna, MG [tese de mestrado] Belo Horizonte (MG): UFMG; 2004

31. Caldeira AP, Goulart EMA. A situação do aleitamento materno em Montes Claros, Minas Gerais: estudo de uma amostra representativa. J Pediatr (Rio J) 2000;76:65-72.

32. Pilviniene R, Maciulaitis R, Jankūnas R, Milvidaite I, Markūniene E. Breastfeeding and medications. Medicina (Kaunas) 2006;42:1035-45.

33. Chaves RG, Lamounier JA, César CC, Corradi MAL, Mello RP, Gontijo CM et al. Amamentação e uso de antiinflamatórios não esteróides pela nutriz: informações científicas versus conteúdo em bulas de medicamentos comercializados no Brasil. Rev Bras Saude Mater Infant 2006;6:269-76.

34. Ito S, Koren G, Einarson TR. Maternal noncompliance with antibiotics during breastfeeding. Ann Pharmacother 1993;27:40-2.

35. Winans EA. Antipsychotics and breastfeeding. J Hum Lact 2001;17:344-7.

36. Matheson I, Kristensen K, Lunde PK. Drug utilization in breast-feeding women. A survey in Oslo. Eur J Clin Pharmacol 1990;38:453-9. 